\title{
Seasonal and Regional Chemical Variability of the Wild Population of Lantana camara Leaf Essential Oil From Kenya
}

\author{
Robai N. Liambila ${ }^{1}$, John M. Wesonga ${ }^{1}$, Catherine N. Ngamau ${ }^{1} \&$ Waudo Wallyambilla ${ }^{2}$ \\ ${ }^{1}$ College of Agriculture Natural Resource, Jomo Kenyatta University of Agriculture and Technology, Nairobi, \\ Kenya \\ ${ }^{2}$ College of Pure Applied Sciences, Jomo Kenyatta University of Agriculture and Technology, Nairobi, Kenya \\ Correspondence: Robai N. Liambila, College of Agriculture Natural Resource, Jomo Kenyatta University of \\ Agriculture and Technology, P.O. Box 62000-00200, Nairobi, Kenya. E-mail: liambilarobai@gmail.com
}

Received: July 1, $2021 \quad$ Accepted: September 25, $2021 \quad$ Online Published: October 15, 2021

doi:10.5539/jas.v13n11p86 URL: https://doi.org/10.5539/jas.v13n11p86

\begin{abstract}
Studies examining the variability in wild plant metabolic expression propose that environmental factors significantly influence the essential oil (EO) quality and quantity in a plant. Lantana camara is a widely distributed invasive plant species worldwide. However, its immense metabolites can become a source of novel compounds to produce biopesticides in the agricultural industry. Although, the quality aspect has to be considered due to the environmental influence on the metabolites synthesised. Therefore, this research aimed to understand the influence of environmental factors and how it shapes the plant's metabolite profile in multiple populations of $L$. camara. Leaf samples were collected from six different geographic regions of Kenya and the corresponding monthly climatic data and soil samples. GC-MS data from leaf EO were analysed with environmental variables (climate and soil data) using unimodally unconstrained and constrained ordination methods for untargeted metabolomics analysis. Partial Least Squares-Discriminant Analysis (PLS-DA) and Random Forests (RF) were used to confirm the variability further. Seasonal and regional variability was observed for secondary metabolites (SMs) in the leaf EO, which correlated to climatic factors and soil attributes. We highlight the season-al-geographic metabolism relationship for L. camara and the combined analytical approach to obtain data that contributes to understanding the influence of environmental factors on the synthesis and accumulation of SMs. This research will have all-embracing implications for maximising phytochemical uniformity.
\end{abstract}

Keywords: adaptation, environmental factors, Lantana camara, seasonality, secondary metabolites

\section{Introduction}

Lantana camara Linn. (Verbenaceae), commonly known as Lantana, is a plant species widely found growing in many parts of the world and produces essential oils (EOs). Lantana is described as an invasive weed in different ecosystems (Bhagwat, Breman, Thekaekara, Thornton, \& Willis, 2012; Willis, 2017), which is related to problems of ecological imbalance in areas infested with this plant because of its ability to adapt to different climate and soil conditions (Aruna \& Balasubramanian, 2015). The composition of the essential oils of $L$. camara collected from several parts of the world are characterised by the principle components being terpenes (monoterpenes and sesquiterpenes) and their oxygenated derivatives (Anjum et al., 2017; Patil, Kumbhar, \& Ambhore, 2017). Cited among the common major constituents identified are the sesquiterpenes, caryophyllene, isocaryophyllene, germacrene D, bicyclogermacrene, caryophyllene oxide, and caryophyllene epoxide (Anjum et al., 2017; Khan, Mahmood, \& Alkhathlan, 2016; Nea et al., 2020, 2017; Omoregie, Aliyu, Doris, Ehiabhi, \& Folashade, 2016; Pereira et al., 2019; Semdé et al., 2018).

Environmental and edaphic factors may influence the production and accumulation of secondary metabolites in plants of the same species growing wild in different regions. The respective group of secondary metabolites plays a significant role in the plant's adaptation to the surrounding environment (Ncube, Finnie, \& Van Staden, 2012; Niinemets, 2015; D. P. Pavarini, S. P. Pavarini, Niehues, \& Lopes, 2012; Pereira et al., 2019; Ramakrishna \& Ravishankar, 2011; Sampaio \& Da Costa, 2018). The environment influences the biosynthesis of secondary metabolites (SMs), facilitating the chemical interaction between plants, leading to the variations in metabolite profile of a plant, therefore, exerting their biological roles as a plastic adaptive response mechanism to their 
environment (Ncube et al., 2012; Pavarini et al., 2012; Pereira et al., 2019; Sampaio \& Da Costa, 2018; Sampaio, Edrada-Ebel, \& Da Costa, 2016).

The metabolites reported from L. camara growing in different regions have shown marked differences in composition and concentration. Several chemotypes have been described, including a sabinene/cineole/b-caryophyllene chemotype from Algeria (Zoubiri \& Baaliouamer, 2012); $\beta$-caryophyllene (9.8\%), 1,8-cineole (9.4\%), and $\beta$-pinene (8.2\%) from Egypt; $\beta$-caryophyllene (23.3\%), $\alpha$-humulene $(11.5 \%)$, germacrene $\mathrm{D}(10.9 \%)$ or davanone $\beta$-caryophyllene/bicyclogermacrene from India (Rana, Prasad, \& Blazquez, $2005)$ and bicyclogermacrene (19.4\%), isocaryophyllene $(16.7 \%)$, valencene $(12.9 \%)$, and germacrene D (12.3\%) from Brazil (Costa et al., 2010). These results have demonstrated that the environmental and edaphic factors influence plays a significant role in producing and accumulating secondary metabolites.

The variation in secondary metabolites' production influenced by environmental conditions can characterise one species' plant populations. In this context, the metabolites may be used as a chemical marker to differentiate species found in specific geographical zones and seasons (De Souza, Ferri, Fiuza, Borges, \& Paula, 2018; Khan et al., 2016; Pereira et al., 2019). Plants that produce essential oils vary considerably in their quality and quantity (composition and concentration of their constituents) due to their interaction with the natural environment.

The vast array of compound synthesis in the L. camara plant points out the adaptive significance for such a diversity of compounds. The variability for the essential oil (EO) composition of $L$. camara can be related to geographical distribution and pressures (Agil \& Hosseinian, 2012; Benites et al., 2009; Javier, Ocampo, Ceballo, \& Javier, 2017; Khan et al., 2016; Murugesan, Senthilkumar, Suresh Babu, \& Rajasugunasekar, 2016; Pereira et al., 2019; Zoubiri \& Baaliouamer, 2012). The metabolism and accumulation of secondary metabolites reflect the integrated influences of multiple ecological factors on the plant during their developmental and growth periods in addition to genetic factors (Liu et al., 2016). Some metabolites are synthesised only under specific environments, or their contents significantly increase under specific environments. Moreover, previous studies have demonstrated that medicinal plants growing in different regions and environments produce different SMs resulting in differences in their qualities (W. Liu, J. Liu, Yin, \& Zhao, 2015). Therefore, studies examining the diversity in the production of SMs of $L$. camara will explain the influence of abiotic and biotic pressures on the EO quality found in a species.

Many studies investigating the influence of environmental factors on plant SM biosynthesis consider these factors' effects on individual compounds. However, individual compounds rarely happen in isolation (Gershenzon, Fontana, Burow, Wittstock, \& Degenhardt, 2012). Instead, any compound's influence depends on conditions within the prevailing environment since a single factor cannot be extrapolated from a combination of environmental factors in plants growing in the natural environment (Berini et al., 2018). Thus, understanding how environmental factors will influence a plant's metabolic profile is vital for interpreting how these changes influence the abundance of individual compounds.

Considering the wide distribution and adaptive capacity of $L$. camara, and the lack of studies covering this subject, as well as the pesticidal properties of this plant, we proposed to carry out a comparative study with samples of EO from leaves of $L$. camara obtained from samples collected from six different climatic zones of Kenya and collected in different seasons. The approach involves comparing the data obtained by chemical profiles of these oils from six regions. To assess the influence of seasonal and geographical location on the production of SMs and determine the underlying factors responsible for the variations in SMs in L. camara, feasibly suggest the best harvesting seasons and regions for this wild species and promote its reasonable exploitation for specific compounds for biopesticide production.

\section{Method}

\subsection{Sampling Locations}

Natural populations of L. camara plant, the first four leaves of the stem from the top (Figure 1A) were sampled from six representative climatic zones (Lower Highland-Njoro (LH-NJ), Upper Midland 1-Kakamega (UM1-KK), Upper Midland 2-Kandara (UM2-KA), Upper Midland 3-Embu (UM3-EM), Lower Midland-Kiboko (LM-KI) and Coastal Lowland-Mtwapa (CL-MT)) located in six counties of Kenya during the wet and dry seasons (2018, 2019 and 2020) (Figure 1B). Each region consisted of four collection sites (each population was separated geographically by at least $30 \mathrm{~km}$ and $5 \mathrm{~m}$ for adjacent individuals).

\subsection{Plant and Soil Material Collections}

The first four leaves of the stem from the top were picked up from four directions (north, south, east, and west), obtaining as many individuals as possible. The leaves from each sampling site were harvested and mixed to 
make a composite sample. The samples were transferred to the lab within two days of collection in nylon gunny bags. The plant samples were passed through running tap water to remove any foreign material and then air-dried immediately under room temperature $\left(23-26^{\circ} \mathrm{C}\right)$ in a well-ventilated room for two weeks until crispy.

Soil samples were collected using an alderman auger at $0-15 \mathrm{~cm}$ depth and $6 \mathrm{~cm}$ diameter directly under $L$. camara plant canopies. Soil samples were placed in labelled plastic bags (four replicates for each geographical location). Soil samples were used to determine the critical soil parameters, including nitrogen (N), phosphorus $(\mathrm{P})$, potassium $(\mathrm{K})$, Total organic carbon (TOC), electrical conductivity (EC) and $\mathrm{pH}$ using the standard soil analysis methods.

Nitrogen $(\% \mathrm{~N})$ was determined using $\mathrm{H}_{2} \mathrm{SO}_{4}$ digestion and measured with the Kjeldahl method. Phosphorus (P) of soil samples was extracted with ammonium fluoride $\left(\mathrm{NH}_{4} \mathrm{~F}, 0.03 \mathrm{M}\right)$ and hydrochloric acid $(\mathrm{HCl}, 0.025 \mathrm{M})$ (Sinopharm Chemical Reagent Co., Ltd, Shanghai, China) and measured by UV-Vis spectrophotometer (Thermo Fisher Scientific, San Jose, CA, USA). Potassium (K) of soil samples was determined by extraction with $\mathrm{CH}_{3} \mathrm{COONH}_{4}(1 \mathrm{M})$ (Sinopharm Chemical Reagent Co., Ltd, Shanghai, China) and quantified by Corning flame photometer (Sherwood Scientific Ltd, Cambridge, UK). Soil pH (soil reaction) was measured in 1:2.5 soil: water suspension solution using the Consort $\mathrm{pH}$ meter model C835. The Electrical Conductivity EC) was measured using the EC meter model 4510 in the soil to water ratio of 1:2.5. The analyses were undertaken at Jomo Kenyatta University of Agriculture and Technology Soil Chemistry Laboratory.

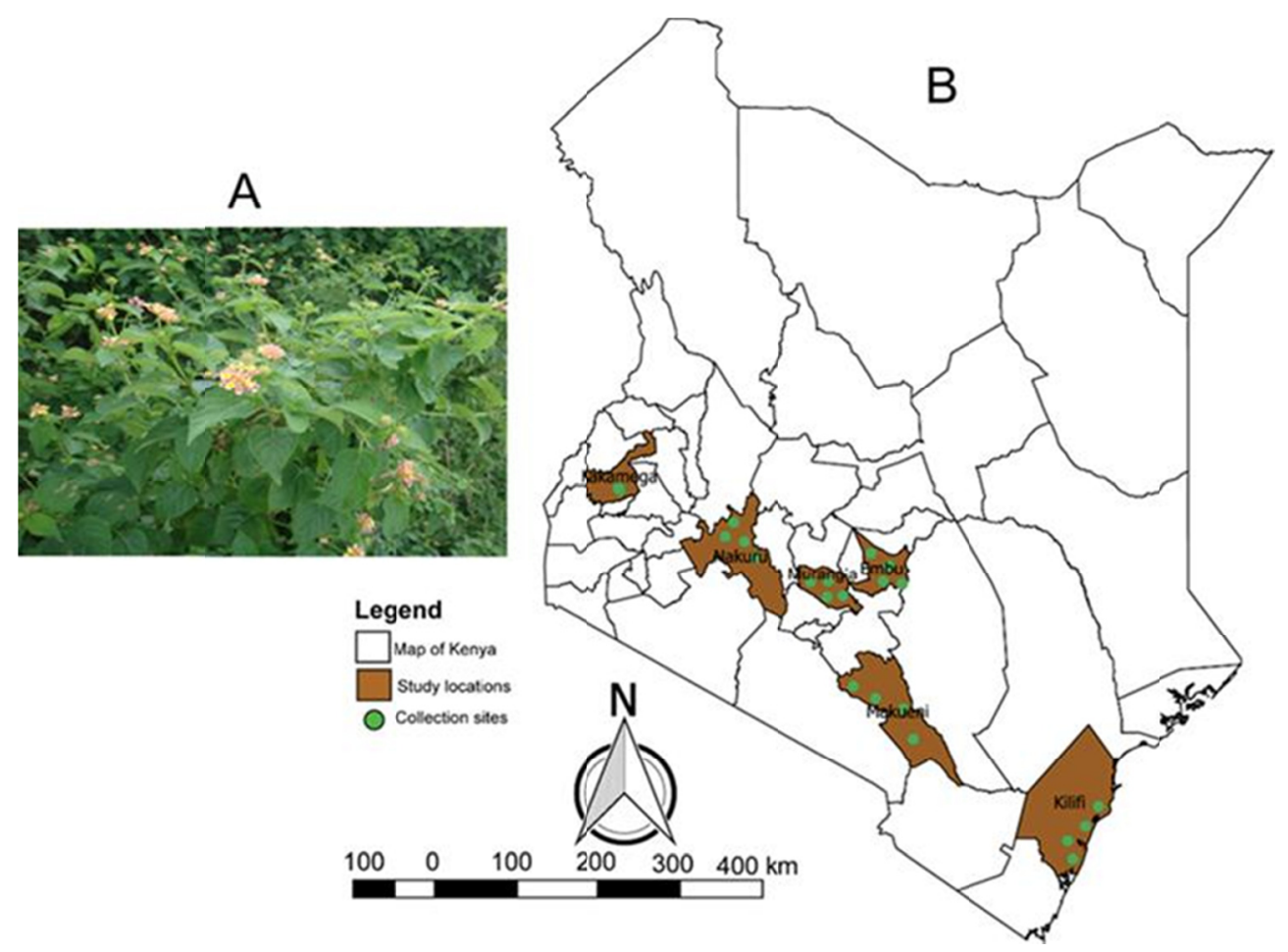

Figure 1. (A) Plant of Lantana camara, (B) Kenyan map showing the different sampling localities

\subsection{Extraction of Volatile Oils}

The volatile oils of $L$. camara were obtained from the dried leaves by steam distillation performed in a steam distiller apparatus (Deschem Science supply, China). $200 \mathrm{~g}$ of each sample was steam distilled using $600 \mathrm{~mL}$ distilled water for $3 \mathrm{hrs}$. The steam, which contains the essential oil, was then passed through a condenser. The condensing oils were separated with a separating funnel and collected in amber-coloured vials, labelled, and stored at $4{ }^{\circ} \mathrm{C}$ until use.

\subsection{Gas Chromatography-Mass Spectrometry (GC-MS) Analysis}

The oil samples were diluted in $100 \% \mathrm{n}$-hexane and transferred to the auto-sampler vials for GC-MS analysis. Agilent Technology (GC, Agilent 6890) (Agilent Technologies Inc., Santa Clara, CA, USA) gas chromatograph with a split detector and Mass Spectrometer Detector (MSD) coupled with an autosampler was used for this 
study. Each sample $(1 \mu \mathrm{L})$ was injected into the GC-MS with an autosampler (Agilent Technologies). The injections of the volatile oil were conducted with a splitless injector at $220{ }^{\circ} \mathrm{C}$. The compounds were then separated on a nonpolar capillary HP column at an average linear flow rate of $35 \mathrm{~cm} \mathrm{~s}^{-1}$ with helium as the carrier gas. The oven temperature was held at $35^{\circ} \mathrm{C}$ for 5 minutes and then increased to $280{ }^{\circ} \mathrm{C}$ by $10{ }^{\circ} \mathrm{C} / \mathrm{min}$ and held for 10 minutes. The collected volatile compounds were then identified by comparing their mass spectra and retention times with the National Institute of Standards and Technology (NIST) 2017 library of mass spectra.

\subsection{Related Data of Climatic Factors}

The environmental data were divided into climate and soil data. The climate data consisted of Monthly average temperature $(\mathrm{aT})$, maximum temperature $(\operatorname{maxT})$ and minimum temperature $(\operatorname{minT})$, average precipitation $(\mathrm{P})$, sunshine duration (SD), and UV index for the collection month were collected from local meteorological stations for the six study sites and were pre-treated in $\mathrm{MS} \mathrm{Excel}^{\circ}$ and used for further analysis.

\subsection{Data Processing and Analysis}

The data for the study of seasonal and geographical variation in the composition and accumulation of SMs of the essential oil of $L$. camara (chemical profiles and environmental data) were divided into two sets of variables: chemical (secondary metabolites (SMs) and environmental (climate and soil data) variables. The data was obtained and used for further analysis after analysing the essential oils by GC-MS (chemical data).

The R software (version 3.6.3; R Core Team, 2020) and the RStudio graphical user interface (version 1.2.5033) were used to perform all the analyses. The total area of peak data was normally distributed (Shapiro-Wilk test: $p>$ 0.05 ), and their variance was homogeneous (Barlet test: $\mathrm{p}>0.05$ ); therefore, we used an unpaired t-test to compare the amount SMs synthesised by L. camara between the rainy and dry seasons. For the same reason, we used the analysis of variance (ANOVA) followed by the Student-Neuman-Keuls (SNK) post hoc test to compare the amount of SM synthesised by $L$. camara across the different localities during a specific season using the $\mathrm{R}$ software package 'Agricolae' (de Mendiburu, 2020). We performed the one-way analysis of similarity (ANOSIM) using the Bray-Curtis dissimilarity matrix to compare the chemical profiles of the different compounds synthesised by $L$. camara between the seasons and across the different localities. Based on the similarity percentage (SIMPER) analysis, we identified the 10 most influential SMs contributing to L. camara EO's diversity between the seasons and across the different localities. To visualise this difference, we first used the non-metric multidimensional scaling (NMDS) plot, overlaid the physicochemical and environmental variables to the plot to see whether these parameters were related to SM profile diversities.

To better confirm the variation of L. camara EO SM composition between seasons and locations, we used two supervised machine learning algorithms, namely: Random Forest (RF) analysis (Breiman, 2001) and Partial Least Squares-Discriminant Analysis (PLS-DA) (Liland \& Indahl, 2009). Helped by the R package called Random Forest (Liaw \& Wiener, 2002), we ran the RF analysis using 10000 iterations (ntree) with 12 SM randomly selected at each split (mtry $=\sqrt{ } \mathrm{q}$, where $\mathrm{q}$ is the total number of SM (150)). Based on the function "importance ()", we generated the mean decrease in accuracy (MDA) for each compound selected. The compound with the highest MDA value was considered the most significant for $L$. camara EO's diversity between the seasons and regions. To visualise these differences, we generated the multidimensional scaling (MDS) ordination plot using the function "MDSplot ()" based on the proximity matrix from the RF analysis. Using the function "PLS-DA ()" embedded in the R package called mixOmics (Rohart, Gautier, Singh, \& Kim-Anh, 2017), we performed the PLS-DA analysis. We visualised the difference using the function "plotIndiv ()". With this technic, we identified the most significant compounds for differentiating L. camara EO using the function "PLS-DA.VIP ()" found in RVAideMemoire R package (Maxime, 2020). All statistical results were considered significant when $\mathrm{P}<0.05$.

\section{Results}

\subsection{Seasonal Variation in Essential Oil Composition of L. camara}

The GC-MS chromatograms from L. camara essential oil showed that the plant synthesised many Secondary Metabolites (SMs) for both rainy and dry seasons (Figure 2). The number of SMs synthesised by L. camara varied significantly between the seasons, except for Kiboko $(\mathrm{p}=0.68$; Figure $2 \mathrm{C})$ and Njoro $(\mathrm{p}=0.64$; Figure 1F). The number of compounds was lower in Mtwapa (Figure 2E) but higher in Embu (Figure 2A), Kandara (Figure 1B) and Kakamega (Figure 2D) during the rainy season. In comparison, there was a tremendous increase in the number of compounds observed in the plants sampled from Embu (Figure 2A) and Mtwapa (Figure 2E) and a reduction in Kandara (Figure 2B) and Kakamega (Figure 2D) during the dry season. Independently to the site, when we ran the analysis of similarity based on the Bray-Curtis distance matrix, we consistently found a 
significant difference of $L$. camara EO SM composition between rainy and dry season (ANOSIM: $\mathrm{p}<0.0001, \mathrm{R}$ $=0.465)$. This difference was depicted by the Non-metric multidimensional Scaling (NMDS) plot (Figure 3A), with an excellent dissimilarity representation (Figure 3B; Stress: 0.199). This plot distinguished L. camara EO obtained during the rainy season from those obtained during the dry season. The similarity percentage (SIMPER) analysis identified trans-cadina-1(6),4-diene, (E-)-Caryophyllene, and 1,8-cineole as the three significant compounds responsible for the distinction of L. camara essential oil between the rainy and dry season (Figure 3C). 

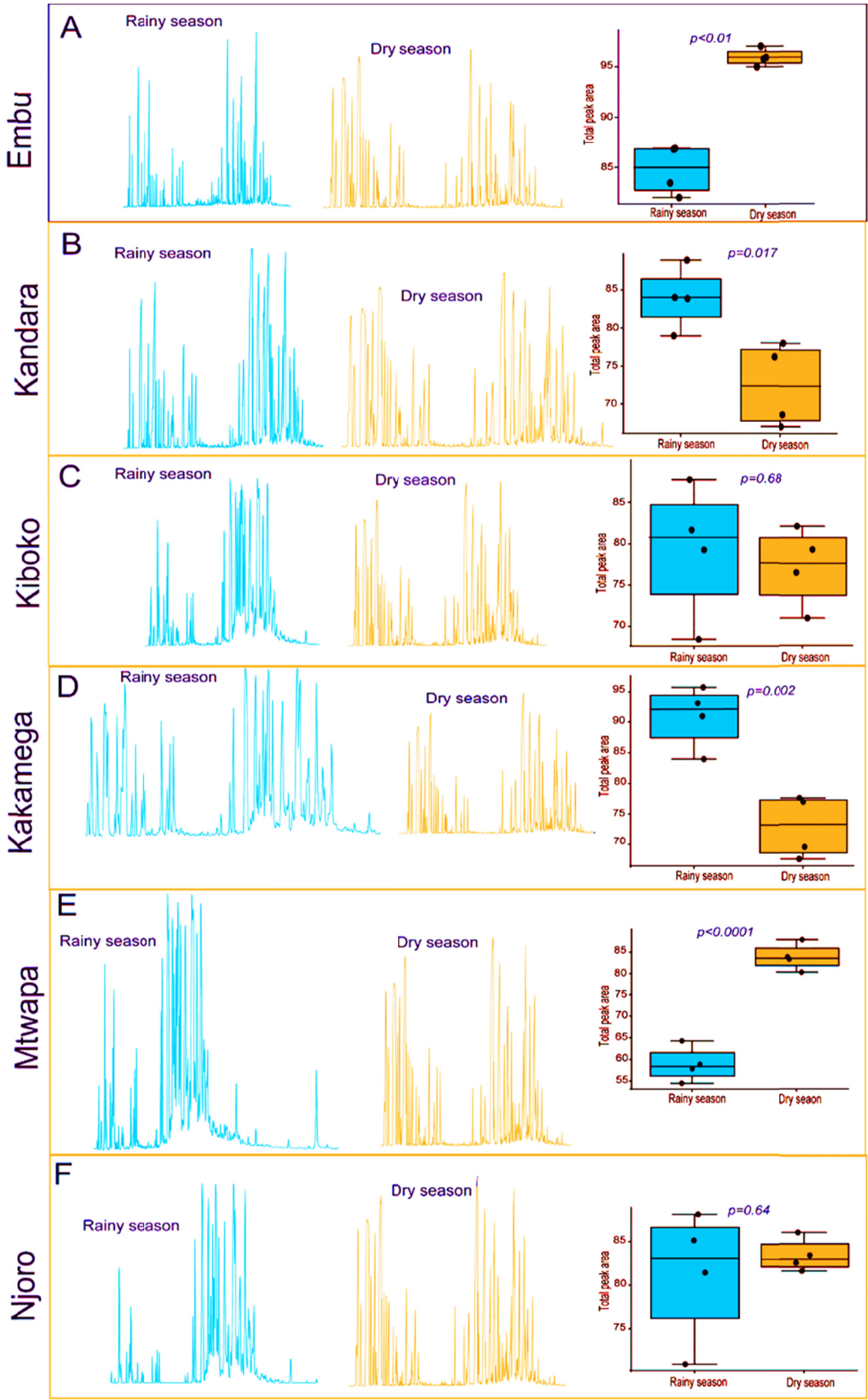

Figure 2. GC-MS chromatogram of L. camara SM synthesised during the rainy (in blue) and dry (in brown) seasons and across the different climatic zones of plant collections. Boxplot depicting the quantitative variation of the total amount of SM synthesised by L. camara between the rainy and dry season in each collection site. The ends of the boxplot whiskers represent the minimum and maximum of all the data 


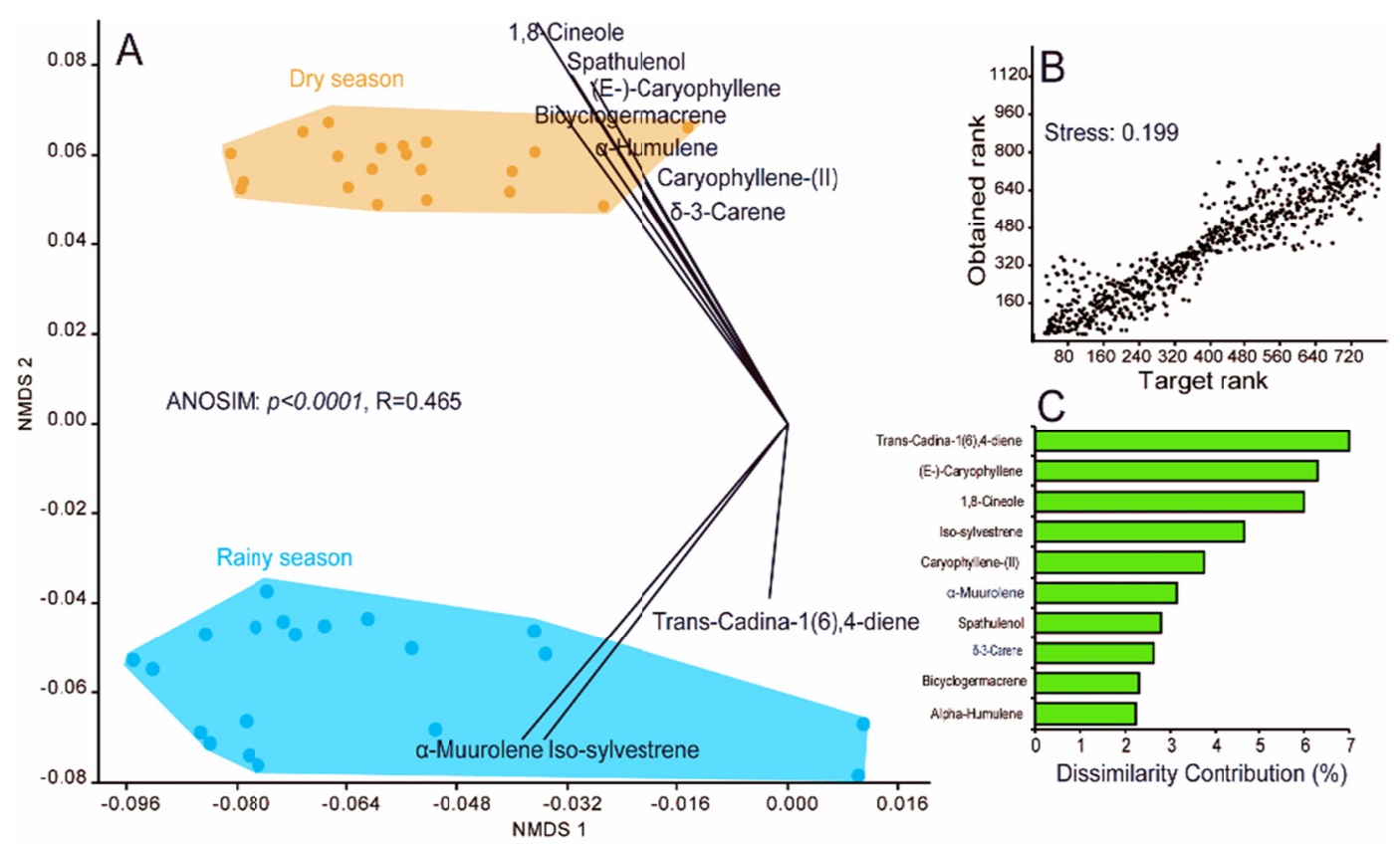

Figure 3. Secondary Metabolites synthesised by Lantana camara varied qualitatively and quantitatively between the rainy and dry seasons. (A) Non-metric multidimensional scaling (NMDS) plot based on Bray-Curtis dissimilarities index illustrates the SM's seasonal variation synthesised by L. camara. (B) Shepard plot showing the quality of the NMDS plot visualisation. (C) Histograms of the first ten most significant compounds contributing to the seasonal variation observed in L. camara essential oil

\subsection{Regional Variation of L. camara Secondary Metabolites During the Rainy Season}

Our precedent result revealed that $L$. camara oil SM composition varied with seasons. Here we aimed to determine whether this composition could also be affected by its location. We found that the total peak area of the SMs synthesised by L. camara significantly differed across the different localities where this plant was collected (Figure 4A). This difference was confirmed by the ANOSIM test and the NMDS plot that clustered $L$. camara SMs in their provenance (Figure 4B). Using the SIMPER analysis, we identified the trans-cadina-1(6),4-diene followed by Caryophyllene-(II), and Lavandulyl isovalerate as the most critical SMs contributing to this chemical variation across the locations (Figure 4D). To see if the observed L. camara EO changes across locations were related to each location's physicochemical and environmental parameters, we overlaid these parameters to the NMDS plot (Figure 4E). We found that the SM's synthesis by L. camara was closely associated with temperature, ultraviolet light (UV), and soil pH in Mtwapa. Simultaneously, in the localities such as Embu, Kiboko, and Kakamega, the synthesis of the SMs was related to the soil parameters such as phosphorous, potassium, and total organic carbon (TOC). While in Njoro, the synthesis of the SMs was more influenced by the humidity. 

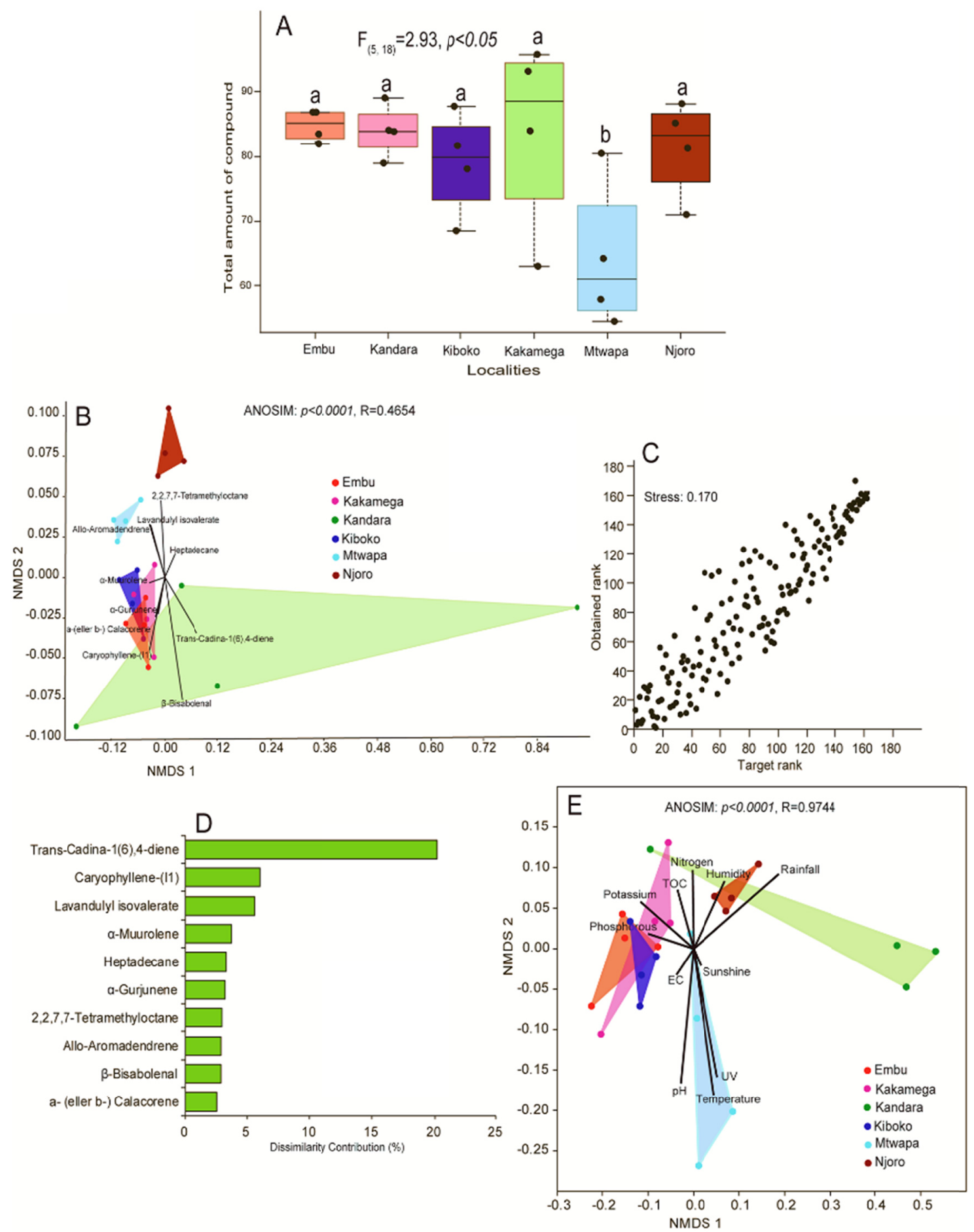

Figure 4. The variation of the SMs observed in L. camara during the rainy season is related to the plant location, the soil's physicochemical properties, and each location's climatic condition. (A) Boxplots showing the variation of the amount of SMs synthesised by L. camara across the different localities. Boxplot whiskers indicate \pm 1.5 interquartile range limits. Boxplots with different letters show significant differences as grouped by ANOVA tests followed by SNK post-hoc tests $(P<0.05)$. (B) Non-metric multidimensional scaling (NMDS) ordination plot illustrating the variation of the SMs synthesised by L. camara across the different localities. (C) Shepard plot assessing the quality of the NMDS plot representation. (D) Histogram depicting the SMs contributing to the overall dissimilarity of the synthesised SMs by L. camara across the different localities. (E) Non-metric multidimensional scaling (NMDS) ordination plot displaying the soil physicochemical and climatic variables that influence the L. camara SMs synthesis in each location 


\subsection{Regional Variation of L. camara EO Secondary Metabolites (SMs) During Dry Season}

We also found qualitative and quantitative variations in L. camara EO across the locations during the dry season. The total SMs synthesised by L. camara significantly varied across the different localities (Figure 5A). L. camara plants sampled from Embu synthesised significantly more SMs than those sampled from Mtwapa, Njoro, Kandara, Kiboko, and Kakamega. According to their origin, there was a significant difference in L. camara EO SM composition as determined by the ANOSIM test and the NMDS plot (Figure 5B). Based on the SIMPER analysis, we identified (E)-Caryophyllene, $\delta$-3-Carene, and 1,8-Cineole as the most critical compounds contributing to $L$. camara differentiation in the dry season across the different (Figure 5D). When we associated the physiochemical and the environmental parameters of each site to the NMDS plot (Figure 5E), we found that the synthesis of SMs by $L$. camara varied from region to region and was primarily related to sunshine, rainfall, humidity, temperature, soil $\mathrm{pH}$, nitrogen, and phosphorous (Figure 5E). 

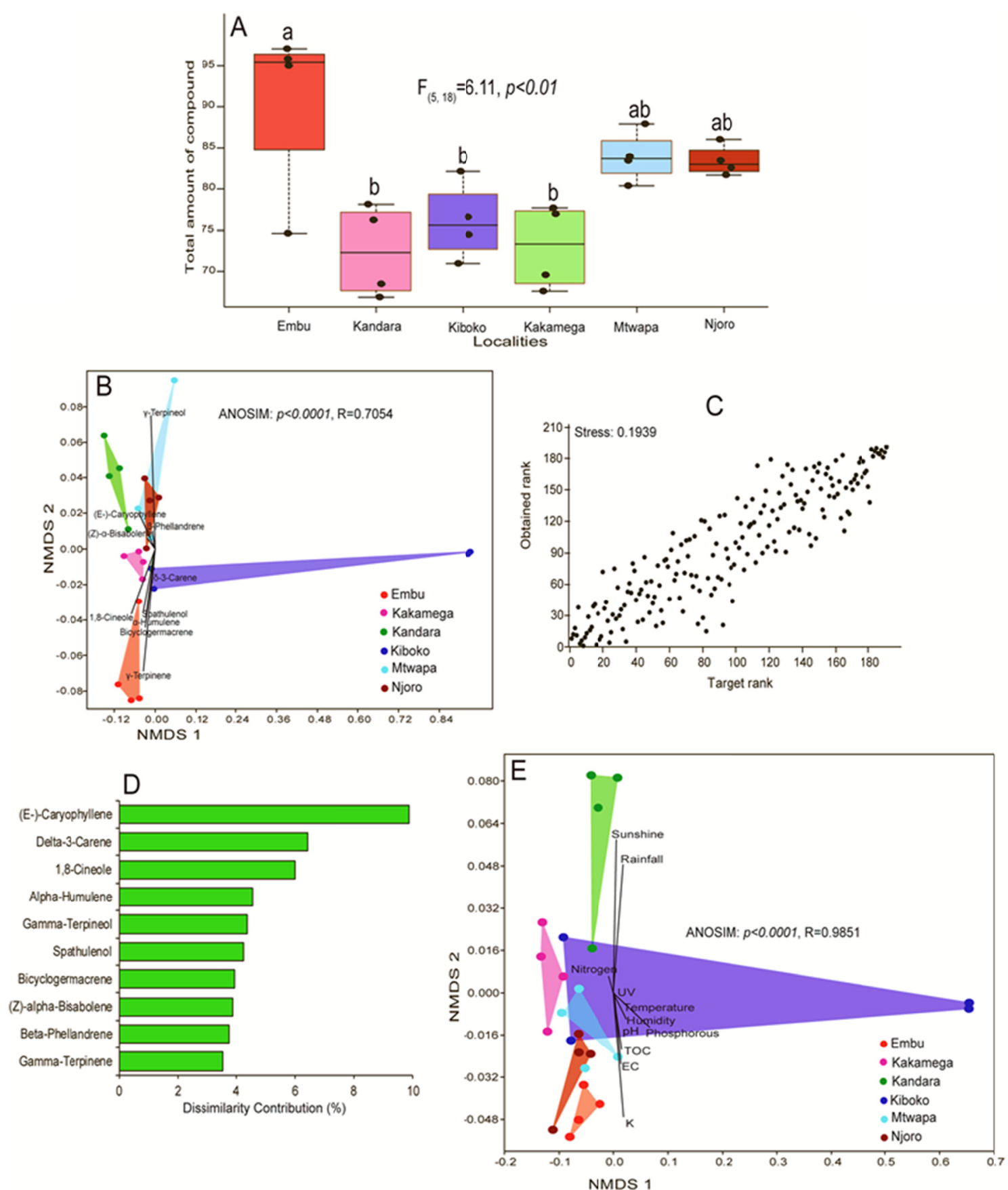

Figure 5. The change in SM observed in L. camara during the dry season is related to the plant location, the soil's physicochemical property, and each location's climatic condition. (A) Boxplots depicting the variation of the amount of SM synthesised by L. camara across the different localities. Each boxplot shows the median (horizontal line), $25^{\text {th }}$ and $75^{\text {th }}$ percentiles (upper and lower box limits). Boxplots with different letters show significant differences as grouped by ANOVA tests followed by SNK post-hoc tests $(P<0.05)$. (B) The non-metric multidimensional scaling (NMDS) ordination plot illustrates the SM synthesised variation by L. camara across the different localities. (C) Shepard plot assessing the quality of the NMDS plot representation. (D) Histogram showing SM that mainly contribute to the overall dissimilarity of $L$. camara EO SM synthesised. (E) Non-metric multidimensional scaling (NMDS) ordination plot displaying the soils physiochemical and the climatic variables that impact the L. camara SM synthesised in each location 
3.4 Confirmation of L. camara SMs Profile Changes in the Function of Seasons and Locations Based on Supervised Machine Learning Algorithms

Results from Random Forest (RF) analysis and Partial Least Squares-Discriminant Analysis (PLS-DA) consistently confirmed the change of L. camara EO SMs in the function of seasons and localities. When we ran the multidimensional scaling analysis based on the RF analysis's proximity matrix, this technic differentiated $L$. camara oil SM based on their seasons (Figure 5Ai) and location (Figure 6Aii, 6Aiii) of collections. Based on the mean decrease in accuracy (MDA), this analysis identified Camphor (with a classification accuracy of 83.33\%) as the most influential SM for differentiating L. camara oil between rainy and dry season (Figure 6Bi). Similarly, this analysis respectively identified 14-hydroxy-(Z)-Caryophyllene (with a classification accuracy of 65\%) and Eugenol (with a classification accuracy of $80 \%$ ) as the most significant SM for distinguishing L. camara oil across the different localities during rainy (Figure 6Bii) and dry (Figure 6Biii) seasons.

Also, the PLS-DA score plots separated L. camara EO in the function of seasons (Figure 7Ai) and locations (Figure 7Aii, iii). As with the RF analysis, the separation of L. camara SM synthesised during the rainy and dry seasons identified Camphor as the most influential compound (Figure 7Bi). The nPLS-DA analysis identified 2-Cyclopenten-1-one, 3-methyl-2-(1,3-pentadienyl)-,(E, Z)- as the most significant SMs responsible for the differentiation of L. camara EO across the localities during rainy (Figure 7Bii) and dry (Figure 7Biii) seasons, respectively. 

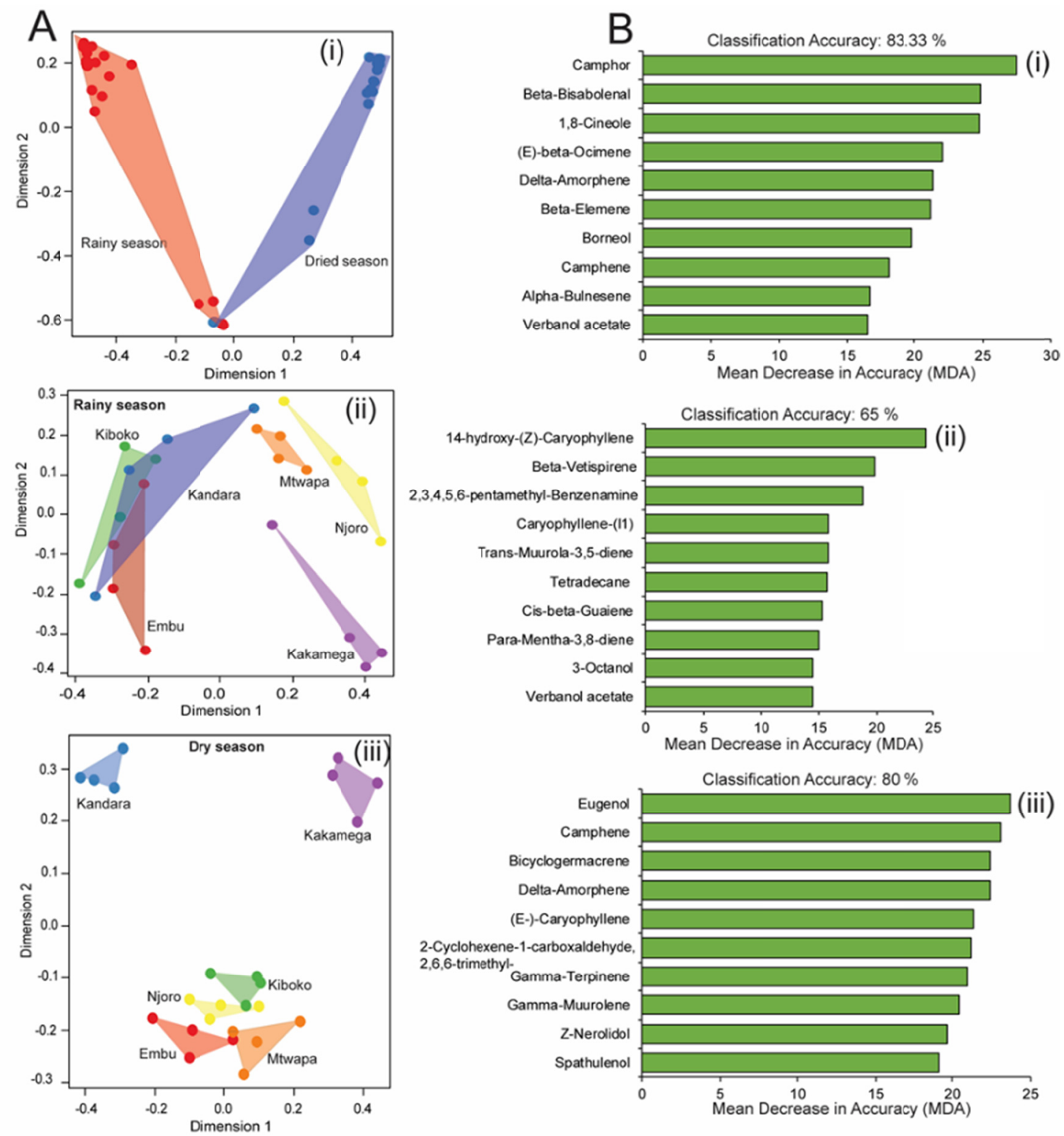

Figure 6. (A) Multidimensional Scaling (MDS) plot based on the proximity matrix coordinates from the Random Forest classification illustrating the change of $L$. camara EO SM synthesised between the seasons (i) and across the different localities during the rainy (ii) and dry (iii) seasons. (B) Histogram showing the most significant SMs contributing to the distinction of $L$. camara EO during rainy and dry seasons (i) and across the different localities during the rainy (ii) and dry seasons (iii) 

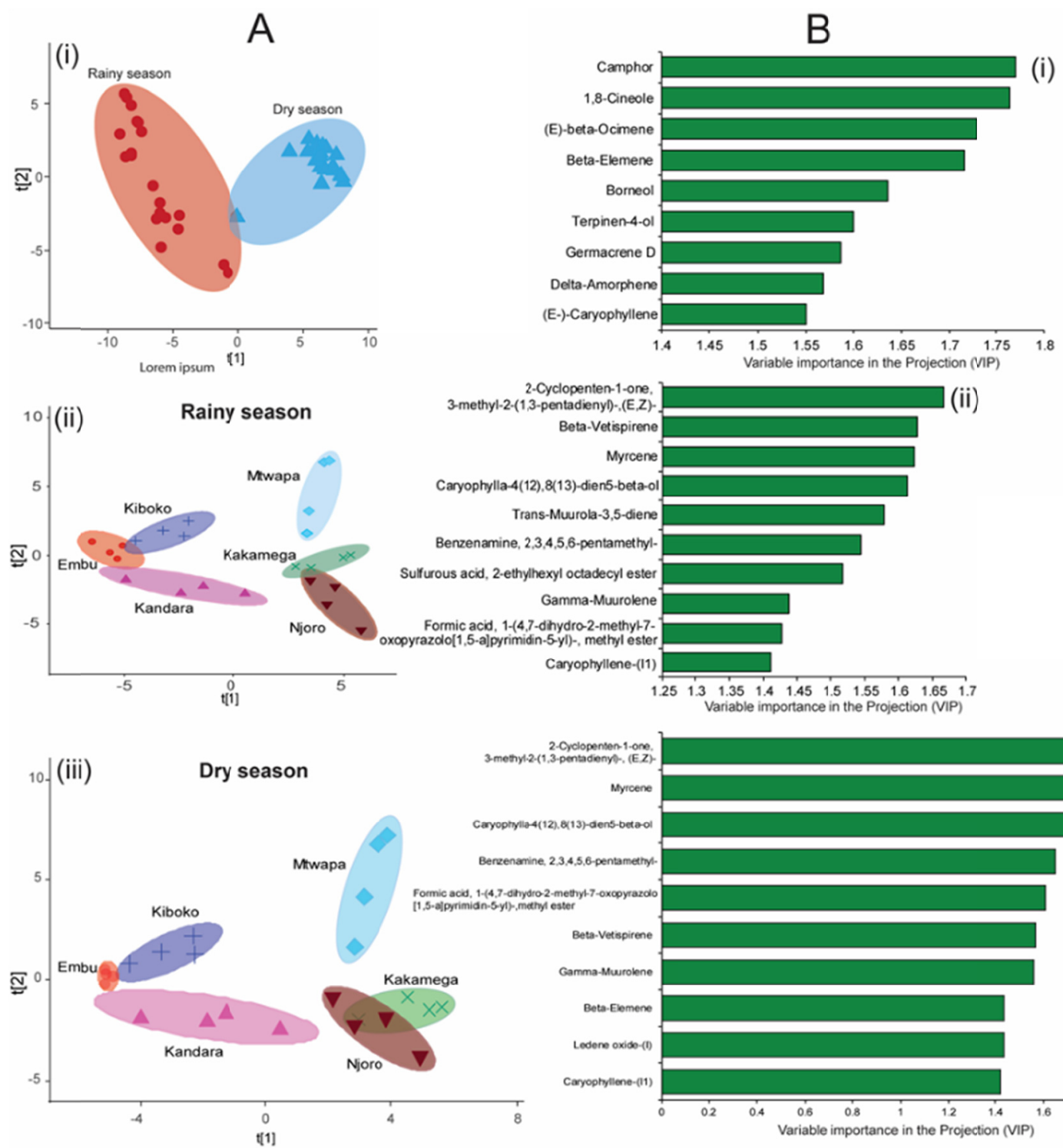

2-0.olopenten-1-one.

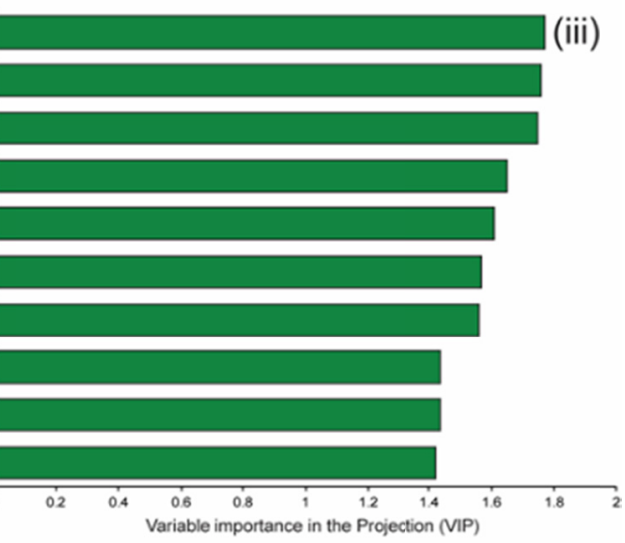

Figure 7. (A) Partial least squares discriminant analysis (PLS-DA) score plot separating the SM synthesised by $L$. camara in the function of seasons (i) and localities during rainy (ii) and dry (iii) seasons. (B) Histograms showing the ten most significant SMs from the PLS-DA model help differentiate SM synthesised by L. camara between the seasons and across the localities during the rainy (ii) and dry (iii) seasons

\section{Discussion}

The natural environment strongly influences the chemo diversity of medicinal plants by influencing the biosynthesis of secondary metabolites (SMs) in a plant population based on the environmental conditions where they are growing (Allevato, Kiyota, Mazzafera, \& Nixon, 2019; Liu et al., 2015; Ncube \& Van Staden, 2015; Pereira et al., 2019; Sampaio et al., 2016). Most plants can regulate SMs according to the growing environment (Isah, 2019; Li, Kong, Fu, Sussman, \& Wu, 2020; Yang et al., 2018), causing changes in the plant's essential oil (EO). Secondary metabolite profiles of plants driven by environmental plasticity are poorly understood. This study established that the production of SMs of the L. camara plant growing in the wild differed significantly during the seasonal changes and geographical location. Therefore, heritable variations observed in the EO of the L. camara wild population across Kenya are strongly linked to climatic factors, geographical location, and soil properties while not disputing the strong influence of the plant species' genetic makeup. Here, we discuss the findings from the ecological point of view. 
Striking variation in the EO of $L$. camara leaf metabolic profile was observed from the samples collected from six different climatic zones in Kenya, dry and rainy seasons. The variability between the $L$. camara plant populations was compared using the ANOSIM test based on differences in the abundance and composition of multiple metabolites. Seasonal specificity is observed in this study, with variability in the number of SMs observed for both dry and rainy season in each region. Kakamega, Kiboko, and Kandara showed an increased metabolites number compared to the other regions in the rainy season. This increase in metabolic variation can be attributed to the plant's interaction with the prevailing condition at that particular season. These regions received adequate rainfall and maximum sunshine hours and UV index compared to the other regions where sunshine was reduced during the rainy seasons. The results agree with other researchers that the metabolite synthesis of essential oil-producing plants is strongly influenced by the slightest changes and how they interact with the natural environment without detrimental effects leading to newly formed compounds that could be advantageous during the prevailing conditions.

Ten dominant variable compounds contributed to shaping the seasonal variation of the $L$. camara plant populations. For the samples of $L$. camara evaluated in this work, we propose two chemotypes according to the season: a chemotype for the samples collected in the dry season (chemotype I) and another for the rainy season (chemotype II). Based on the NMDS test results and SIMPER analysis, the chemotype I is discriminated by the higher proportion of (E-)-caryophyllene, 1,8-cineole, spathulenol bicyclogermacrene, $\alpha$-humulene, caryophyllene (II), and $\delta$-3-carene in the leaf EO. The chemotype II is discriminated by the higher proportion of trans-cadina-1(6),4-diene, $\alpha$-muurolene, and Iso-sylvestrene, as the dominant compounds. Overall, three significant compounds, trans-cadina-1(6),4-diene, (E-)-Caryophyllene, and 1,8-cineole, contributed significantly to the seasonal variability in the SM profile of the L. camara EO.

The environmental factors are the mechanism involved in influencing the accumulation and biosynthesis of $L$. camara SMs, and it is related to seasonal induction stress factors in the plant, such as changes in temperatures, humidity, rainfall and soil conditions (Guo et al., 2013; Pavarini et al., 2012; Ramakrishna \& Ravishankar, 2011; Selmar \& Kleinwächter, 2013; Yang et al., 2018). For example, plants collected during the dry season may have encountered high temperatures influencing an increase in (E-)-caryophyllene production, while low temperatures in the rainy season influenced an increase in trans-cadina-1(6),4-diene. This result agrees with De Almeida et al. (2016), who reported an increase in (E-)-caryophyllene in the EO composition of Copaifera langsdorffii during the dry season as compared to the wet season. There is no official report in the literature regarding the influence of seasonal change on trans-cadina-1(6),4-diene accumulation. This variability can be attributed to the plants' relationship with the environmental conditions during plant growth and productivity at that particular season, changing the biosynthetic pathway of secondary metabolites toward metabolites' production. Therefore, different prevailing environmental pressures between the two seasons played a significant role in the diversity of the SMs of L. camara leaf EO. Our results corroborate with Nea et al. (2020), Pereira et al. (2019) and Dos Santos et al. (2019) reported fluctuation patterns of SMs produced by L. camara to correlate to seasonal changes. Collectively these results revealed that the seasonal variation predominantly influences the SM profile composition in wild plant populations. Therefore, this work provides new insight into understanding the response of $L$. camara plant populations SMs biosynthesis to the seasonal variation.

Our precedent results revealed seasonal variability in the synthesis of SM in the L. camara wild population plants. We further aimed to determine whether this variability is regional and what environmental factors drive the variation. This study observed significant variability in L. camara EO's chemical profile across the different regions where samples were collected during the dry and rainy season as determined by the ANOSIM test and NMDS plot. Three significant compounds trans-cadina-1(6),4-diene, caryophyllene (II) and lavandulyl isovalerate were identified that contribute significantly to the regional variability in the rainy season, while (E-)-Caryophyllene, $\delta$-3-carene and 1,8-cineole contributed to the differentiation during the dry season across the regions. These results are consistent with previous reports (Murugesan et al., 2016; Pereira et al., 2019; Sena Filho et al., 2012). Recently, Nea et al. (2020), and Pereira et al. (2019) confirmed high variability in SM composition of the EO of L. camara from Bregbo South of Cote d'Ivoire and samples collected from 21 municipalities representing three regions in Brazil respectively. Geographical location is a crucial factor that affects plant growth's prevailing conditions, having significant effects on secondary metabolic processes in a plant species (Liu et al., 2015) - Consequently, the cause of the relationship between the SM composition and contents in their growing locations.

The number of synthesised compounds variability across the regions in both seasons were more or less the same in the region of Njoro and Kiboko with a p-value of 0.64 and 0.68 respectively but differed significantly in Mtwapa, Embu, Kandara, and Kakamega with a p-value of $<0.0001,<0.01,0.017$ and 0.002 respectively. Overall, Mtwapa 
showed high variability in the number of compounds synthesised as compared to the other regions. The number of compounds synthesised was very high during the dry season as compared to the rainy season. The significant difference in SM content is climatic factors of temperature, rainfall, UV index, and $\mathrm{pH}$ property. These changes in the environmental conditions may explain the differentiation of SMs in the Mtwapa region, located in the coastal areas and dominated with a more considerable climatic seasonal variability, particularly temperature, compared to Njoro and Kiboko that had a reasonably stable climatic condition. Temperature change is known to substantially affect SM synthesis since areas with more considerable climatic changes are faced with more variation and could lead to more significant variability in their SM profile (Allevato et al., 2019). Furthermore, Molina-Montenegro and Naya (Molina-Montenegro \& Naya, 2012) argue that locations with slight seasonal variations and constant warm temperatures lead to a low environmental plasticity capacity. Subsequently, stability in the environment would reduce the plant's overall pressures, therefore reducing metabolite variation.

The significant differences in climatic conditions and soil characteristics among the six regions and other explanations are the determinant factors for the variability in SM profile observed in L. camara EO of the same species growing in Kenya's diverse regions. It is observed that $L$. camara wild plants population responds differently to environmental variations, therefore variability in the production and accumulation of SMs. Ncube et al. (2012) explain that these variabilities are due to the physiological characteristics associated with genetic conditions, which arise probably with the prevailing climate in both seasons in the regions under study. Plant metabolism is influenced in many ways by those conditions. Factors such as temperature, humidity, rainfall, sunshine duration directly respond to these variations. These factors jointly influence the biosynthesis and accumulation of SMs and correlate with each other and do not act in isolation (Gobbo-Neto \& Lopes, 2007).

Among the environmental conditions, soil characteristics represent a complicated biological system that strongly influences the plant's ability to produce SMs (Muscolo et al., 2019; Ramakrishna \& Ravishankar, 2011). The nutritional elements (e.g., N, P, K) of soil are required for medicinal plants' growth and are actively involved in plants' metabolic activities (Al-Humaid, 2005; Chrysargyris, Xylia, Botsaris, \& Tzortzakis, 2017; Muscolo et al., 2019; Yadegari, 2015). Correctly, soil characteristics play a crucial role in diverse soil conditions that causes significant differences in biosynthesis and SM accumulation in plants of the same species. The SM profile variation response of $L$. camara to the soils with diverse characteristics was in agreement with the findings of Ormeño and Fernandez (2012), and Muscolo et al. (2019), showing that soil, with its intrinsic characteristics, is directly responsible for plant metabolite production.

This study established a relationship between soil properties and metabolic profile to be regionally specific. Extrapolating these results at the regional and seasonal scale suggests that any alteration of the soil properties leads to changes in SMs accumulation in the L. camara plant population, affecting its quality and quantity. The plant material analysed in this study was collected from plants growing under diverse natural conditions. Therefore, it was not easy to separate the effects of individual factors from the environment's multifactorial influence (Climatic and soil variables). Therefore, we conclude that different climatic factors (rainfall, temperature, UV, humidity) have different effects and intensities on the accumulation of SMs in the EO of L. camara. At the same time, different SMs are affected by different kinds of soil properties in the soil to a different extent, where the physicochemical properties including $\mathrm{N}, \mathrm{P}, \mathrm{K}, \mathrm{TOC}, \mathrm{EC}$ and $\mathrm{pH}$ in the soil all have a relatively significant strong effect on plant secondary metabolism. Thus the correlation differs significantly with SM composition from region to region.

Besides, further identifying the compounds through PLS-DA and RF confirmed SMs variability as a season and geographical location. The results indicated that SMs composition and contents were near related to the growing locations of L. camara, and environmental factors influenced the production of the SMs. The SMs synthesis and accumulation in medicinal plants are complex processes affected by many environmental factors comprising a multivariate system. The variability in chemical profile was expected because the soil factors were significantly different due to the different growing regions and the climatic factors changed with geographical conditions. Local adaptation depends on both genetic, soil properties and environmental factors; Thus, the high chemical variability reflects the need for the plant to adapt to such different environments (Ncube et al., 2012; Pereira et al., 2019; Sampaio \& Da Costa, 2018; Sampaio et al., 2016).

This difference observed could lead to a more targeted analysis and understanding of biological pathways. Although determining the plasticity of a species is difficult without a conventional garden experiment. Our study used only ordination methods to associate climatic and soil variables with variation in chemical profiles of $L$. camara, and correlation does not imply a relationship. Many laboratory and greenhouse experiments are needed to confirm the effect of climatic and soil variables on the SM profile. Greenhouse experiments with the different species in the exact location could help detect whether chemical variation differences are due to environmental, 
soil, or genetic differences. The result would be beneficial for the production of EO with unique active ingredients. One could choose the location of plant growth for a particular compound, or one could add/omit nutrients to modify the EO quality and quantity. Therefore this exploratory analysis in wild populations of L. camara is advantageous and essential as it has reduced the environmental and soil variables and will allow for a more guided experimental analysis such as greenhouse experiments.

\section{Conclusions}

This study provides information on seasonal and regional variations of the quality and quantity of SMs of $L$. camara EO from the leaves. The results showed that environmental conditions in the drier season favour the production of more dominant compounds than in the rainy season. The optimum time to achieve the highest quantity of (E-) caryophyllene is during the drier period, while the trans-cadina-1(6), 4-diene is more abundant in rainy seasons. We can highlight the relationship between the environment and the metabolic profile of $L$. camara, and the variation in SMs is a direct response to fluctuations in conditions in the surrounding environment. Therefore, the knowledge gathered from this study on the influence of seasonal and regional variation on $L$. camara EO composition can help decide the best period to harvest the plant according to the desired compound for exploitation in the agricultural industry. The analysis of wild populations of $L$. camara has provided us with potential environmental variables that require more follow up with greenhouse experiments to determine their importance in SM biosynthesis.

\section{References}

Agil, R., \& Hosseinian, F. S. (2012). Bioactivity of alkylresorcinols. Bioactive Molecules in Plant Foods, 72(December), 131-162.

Al-Humaid, A. I. (2005). Effects of Compound Fertilisation on Growth and Alkaloids of Datura Plants. Journal of Plant Nutrition, 27(12), 2203-2219. https://doi.org/10.1081/pln-200034685

Allevato, D. M., Kiyota, E., Mazzafera, P., \& Nixon, K. C. (2019). Ecometabolomic analysis of wild populations of Pilocarpus pennatifolius (Rutaceae) using unimodal analyses. Frontiers in Plant Science, 10, 258. https://doi.org/10.3389/fpls.2019.00258

Anjum, A., Saima, T., Syeda, Q. Z., Sabira, B., Shaheen, B., Bina, S. S., \& Aqeel, A. (2017). Antimicrobial activity of Lantana camara Linn. 1,3. FUUAST Journal of Biology, 7(January), 127-130.

Aruna, R., \& Balasubramanian, P. (2015). Fruiting Phenology and Avian Frugivory of Streblus asper Lour. in a Mixed Dry Deciduous Forest, Western Ghats, India. International Letters of Natural Sciences, 22(4), 16-21. https://doi.org/10.18052/www.scipress.com/ilns.22.16

Benites, J., Moiteiro, C., Miguel, G., Rojo, L., López, J., Venâncio, F., ... Torres, I. (2009). Composition and biological activity of the essential oil of peruvian Lantana camara. Journal of the Chilean Chemical Society, 54(4), 379-384. https://doi.org/10.4067/S0717-97072009000400012

Berini, J. L., Brockman, S. A., Hegeman, A. D., Reich, P. B., Muthukrishnan, R., Montgomery, R. A., ... Esteban, R. (2018). Combinations of Abiotic Factors Differentially Alter Production of Plant Secondary Metabolites in Five Woody Plant Species in the Boreal-Temperate Transition Zone. Front Plant Sci, 9, 1257. https://doi.org/10.3389/fpls.2018.01257

Bhagwat, S. A., Breman, E., Thekaekara, T., Thornton, T. F., \& Willis, K. J. (2012). A Battle Lost ? Report on Two Centuries of Invasion and Management of Lantana camara L . in Australia, India and South Africa, 7(3), 1-10. https://doi.org/10.1371/journal.pone.0032407

Breiman, L. (2001). ST4_Method_Random_Forest. Machine Learning, 45(1), 5-32. https://doi.org/10.1017/ CBO9781107415324.004

Chrysargyris, A., Xylia, P., Botsaris, G., \& Tzortzakis, N. (2017). Antioxidant and antibacterial activities, mineral and essential oil composition of spearmint (Mentha spicata L.) affected by the potassium levels. Industrial Crops and Products, 103, 202-212. https://doi.org/10.1016/j.indcrop.2017.04.010

Costa, J. G., Rodrigues, F. O., Sousa, E. M. S., Junior, D., Campos, A., Coutinho, H., \& de Lima, S. (2010). Composition and larvicidal activity of the essential oils of Lantana camara and Lantana montevidensis. Chemistry of Natural Compounds, 46(2), 313-315. https://doi.org/10.1007/s10600-010-9601-x

De Almeida, L. F. R., De Portella, R. O., Bufalo, J., Marques, M. O. M., Facanali, R., \& Frei, F. (2016). Non-Oxygenated sesquiterpenes in the essential oil of copaifera langsdorffii desf. Increase during the day in the dry season. PLoS ONE, 11(2), 1-12. https://doi.org/10.1371/journal.pone.0149332 
de Mendiburu, F. (2020). agricolae: Statistical Procedures for Agricultural Research (R Package Version 1.3-2). Retrieved from https://cran.r-project.org/package $=$ agricolae

De Souza, S. J. O., Ferri, P. H., Fiuza, T. S., Borges, L. L., \& Paula, J. R. (2018). Chemical composition and seasonality variability of the Spiranthera odoratissima volatile oils leaves. Brazilian Journal of Pharmacognosy, 28(1), 16-20. https://doi.org/10.1016/j.bjp.2017.10.010

Dos Santos, R. C., De Melo Filho, A. A., Chagas, E. A., Fernández, I. M., Takahashi, J. A., \& Ferraz, V. P. (2019). Influence of diurnal variation in the chemical composition and bioactivities of the essential oil from fresh and dried leaves of Lantana camara. Journal of Essential Oil Research, 31(3), 228-234. https://doi.org/ 10.1080/10412905.2018.1555102

Gershenzon, J., Fontana, A., Burow, M., Wittstock, U., \& Degenhardt, J. (2012). Mixtures of plant secondary metabolites: metabolic origins and ecological benefits. The Ecology of Plant Secondary Metabolites: From Genes to Global Processes (p. 335). Cambridge University Press, UK. https://doi.org/10.1017/CBO9780 511675751

Gobbo-Neto, L., \& Lopes, N. P. (2007). Plantas medicinais: Fatores de influência no conteúdo de metabólitos secundários. Quimica Nova, 30(2), 374-381. https://doi.org/10.1590/S0100-40422007000200026

Guo, L. P., Wang, S., Zhang, J., Yang, G., Zhao, M. X., Ma, W. F., .. Huang, L. Q. (2013). Effects of ecological factors on secondary metabolites and inorganic elements of Scutellaria baicalensis and analysis of geoherblism. Science China Life Sciences, 56(11), 1047-1056. https://doi.org/10.1007/s11427-013-4562-5

Isah, T. (2019). Stress and defense responses in plant secondary metabolites production. Biological Research, 52(1), 39. https://doi.org/10.1186/s40659-019-0246-3

Javier, A. M. V, Ocampo, V. R., Ceballo, F. A., \& Javier, P. A. (2017). Insecticidal Activity of Selected Essential Oil Extracts Against Common Cutworm, Spodoptera litura Fabricius (Lepidoptera: Noctuidae). Philipp Ent 32(2), 117-132.

Khan, M., Mahmood, A., \& Alkhathlan, H. Z. (2016). Characterisation of leaves and flowers volatile constituents of Lantana camara growing in central region of Saudi Arabia. Arabian Journal of Chemistry, 9(6), 764-774. https://doi.org/10.1016/j.arabjc.2015.11.005

Li, Y., Kong, D., Fu, Y., Sussman, M. R., \& Wu, H. (2020). The effect of developmental and environmental factors on secondary metabolites in medicinal plants. Plant Physiology and Biochemistry, 148(January), 80-89. https://doi.org/10.1016/j.plaphy.2020.01.006

Liaw, A., \& Wiener, M. (2002). Classification and Regression by randomForest. $R$ News, 2, 18-22.

Liland, K. H., \& Indahl, U. G. (2009). Powered partial least squares discriminant analysis. Journal of Chemometrics, 23(1), 7-18. https://doi.org/10.1002/cem.1186

Liu, W., Dongxue, Y., Li, N., Xiaogai, H., Dongmei, W., Li, D., \& Liu, J. (2016). Influence of Environmental Factors on the Active Substance Production and Antioxidant Activity in Potentilla fruticosa L. and Its Quality Assessment. Scientific Reports, 6, Article Number 28591. https://doi.org/10.1038/srep28591

Liu, W., Liu, J., Yin, D., \& Zhao, X. (2015). Influence of ecological factors on the production of active substances in the anti-cancer plant Sinopodophyllum hexandrum (Royle) T.S. Ying. PLoS ONE, 10(4), 1-22. https://doi.org/10.1371/journal.pone.0122981

Maxime, H. (2020). RVAideMemoire: Testing and Plotting Procedures for Biostatistics (R Package Version 0.9-75). Retrieved from https://cran.r-project.org/package=RVAideMemoire

Molina-Montenegro, M. A., \& Naya, D. E. (2012). Latitudinal Patterns in Phenotypic Plasticity and Fitness-Related Traits: Assessing the Climatic Variability Hypothesis $(\mathrm{CVH})$ with an Invasive Plant Species. PLoS ONE, 7(10), 23-28. https://doi.org/10.1371/journal.pone.0047620

Murugesan, S., Senthilkumar, N., Suresh Babu, D., \& Rajasugunasekar, D. (2016). Chemical constituents and toxicity assessment of the leaf oil of Lantana camara Linn from Tamilnadu regions. Asian Journal of Plant Science and Research, 6(3), 32-42. Retrieved from http://www.imedpub.com/articles/chemical-constituentsand-toxicity-assessment-of-the-leaf-oil-oflantana-camara-linn-from-tamilnadu-regions.pdf

Muscolo, A., Sidari, M., Settineri, G., Papalia, T., Mallamaci, C., \& Attinà, E. (2019). Influence of Soil Properties on Bioactive Compounds and Antioxidant Capacity of Brassica rupestris Raf. Journal of Soil Science and Plant Nutrition, 19(4), 808-815. https://doi.org/10.1007/s42729-019-00080-5 
Ncube, B., \& Van Staden, J. (2015). Tilting plant metabolism for improved metabolite biosynthesis and enhanced human benefit. Molecules, 20(7), 12698-12731. https://doi.org/10.3390/molecules200712698

Ncube, B., Finnie, J. F., \& Van Staden, J. (2012). Quality from the field: The impact of environmental factors as quality determinants in medicinal plants. South African Journal of Botany, 82, 11-20. https://doi.org/ 10.1016/j.sajb.2012.05.009

Nea, F., Albert, D., Genva, M., Tanoh, E. A., Wognin, E. L., Martin, H., ... Fauconnier, M. (2020). Composition, Seasonal Variation, and Biological Activities of Lantana camara Essential Oils from Côte d'Ivoire. Molecules, 25(10), 2400. https://doi.org/10.3390/molecules25102400

Nea, F., Tanoh, E. A., Yapi, T. A., Garcia, G., Tomi, F., \& Tonzibo, Z. F. (2017). Chemical investigation on leaf, flower and fruit oils of Lantana camara from Côte d'Ivoire. Natural Product Communications, 12(4), 607-610. https://doi.org/10.1177/1934578x1701200434

Niinemets, Ü. (2015). Uncovering the hidden facets of drought stress: Secondary metabolites make the difference. Tree Physiology, 36(2), 129-132. https://doi.org/10.1093/treephys/tpv128

Omoregie, E., Aliyu, I., Doris, E., Ehiabhi, O., \& Folashade, K. (2016). Chemical Components of the Essential Oil of Lantana camara L. Found in North Central Sudan Region of Nigeria. American Chemical Science Journal, 12(1), 1-7. https://doi.org/10.9734/acsj/2016/22944

Ormeño, E., \& Fernandez, C. (2012). Effect of Soil Nutrient on Production and Diversity of Volatile Terpenoids from Plants. Current Bioactive Compounds, 8(1), 71-79. https://doi.org/10.2174/157340712799828188

Patil, S. P., Kumbhar, S. T., \& Ambhore, V. (2017). Evaluation of Unsaponified Petroleum Ether Extract of Lantana camara L. leaves for Antioxidant Activity and Oxidative Stability. Indian Journal of Pharmaceutical Education and Research, 51(4), 692-699. https://doi.org/10.5530/ijper.51.4.102

Pavarini, D. P., Pavarini, S. P., Niehues, M., \& Lopes, N. P. (2012). Exogenous influences on plant secondary metabolite levels. Animal Feed Science and Technology, 176(1-4), 5-16. https://doi.org/10.1016/j.anifeedsci. 2012.07.002

Pereira, K. L. G., Nogueira, P. C. D. L., Arrigoni-Blank, M. D. F., Nizio, D. A. D. C., Silva, D. C., Pinto, J. A. O., ... Blank, A. F. (2019). Chemical diversity of essential oils of Lantana camara L. native populations. Journal of Essential Oil Research, 00(00), 1-16. https://doi.org/10.1080/10412905.2019.1661879

R Core Team. (2020). R: A Language and Environment for Statistical Computing. Vienna: R Foundation for Statistical Computing.

Ramakrishna, A., \& Ravishankar, G. A. (2011). Influence of abiotic stress signals on secondary metabolites in plants. Plant Signaling and Behavior, 6(11), 1720-1731. https://doi.org/10.4161/psb.6.11.17613

Rana, V. S., Prasad, D., \& Blazquez, M. A. (2005). Chemical composition of the leaf oil of Lantana camara. Journal of Essential Oil Research, 17(2), 198-200. https://doi.org/10.1080/10412905.2005.9698874

Rohart, F., Gautier, B., Singh, A., \& Kim-Anh, L. C. (2017). mixOmics: An R package for 'omics feature selection and multiple data integration. PLoS Comput Biol., 13(11), e1005752. https://doi.org/10.1371/ journal.pcbi.1005752

Sampaio, B. L., \& Da Costa, F. B. (2018). Influence of abiotic environmental factors on the main constituents of the volatile oils of Tithonia diversifolia. Brazilian Journal of Pharmacognosy, 28(2), 135-144. https://doi.org/10.1016/j.bjp.2018.02.005

Sampaio, B. L., Edrada-Ebel, R., \& Da Costa, F. B. (2016). Effect of the environment on the secondary metabolic profile of Tithonia diversifolia: A model for environmental metabolomics of plants. Scientific Reports, 6(July), 1-11. https://doi.org/10.1038/srep29265

Selmar, D., \& Kleinwächter, M. (2013). Stress enhances the synthesis of secondary plant products: The impact of stress-related over-reduction on the accumulation of natural products. Plant and Cell Physiology, 54(6), 817-826. https://doi.org/10.1093/pcp/pct054

Semdé, Z., Koudou, J., Figueredo, G., Zongo, C., Somda, K. M., Sawadogo Lingani, H., \& Traoré, S. A. (2018). Chemical composition, antioxidant and antimicrobial activities of Lantana camara Linn leaves essential oil from Burkina Faso. GSC Biological and Pharmaceutical Sciences, 5(3), 124-135. https://doi.org/10.30574/ gscbps.2018.5.3.0142 
Sena Filho, J. G. de, Rabbani, A. R. C., dos Santos Silva, T. R., da Silva, A. V. C., Souza, I. A., Santos, M. J. B. A., ... Duringer, J. M. (2012). Chemical and molecular characterisation of fifteen species from the Lantana (Verbenaceae) genus. Biochemical Systematics and Ecology, 45, 130-137. https://doi.org/10.1016/ j.bse.2012.07.024

Willis, K. J. (2017). State of the World's Plants 2017 (p. 84). Royal Botanic Gardens, Kew.

Yadegari, M. (2015). Foliar application of micronutrients on essential oils of borago, thyme and marigold. Journal of Soil Science and Plant Nutrition, 15(4), 949-964. https://doi.org/10.4067/S0718-9516201500 5000066

Yang, L., Wen, K. S., Ruan, X., Zhao, Y. X., Wei, F., \& Wang, Q. (2018). Response of plant secondary metabolites to environmental factors. Molecules, 23(4), 1-26. https://doi.org/10.3390/molecules23040762

Zoubiri, S., \& Baaliouamer, A. (2012). Chemical composition and insecticidal properties of Lantana camara L. leaf essential oils from Algeria. Journal of Essential Oil Research, 24(4), 377-383. https://doi.org/10.1080/ 10412905.2012.692910

\section{Copyrights}

Copyright for this article is retained by the author(s), with first publication rights granted to the journal.

This is an open-access article distributed under the terms and conditions of the Creative Commons Attribution license (http://creativecommons.org/licenses/by/4.0/). 